\title{
Erratum: Spin-Dependent Weakly-Interacting-Massive-Particle-Nucleon Cross Section Limits from First Data of PandaX-II Experiment [Phys. Rev. Lett. 118, 071301 (2017)]
}

\author{
Changbo Fu et al. \\ (PandaX-II Collaboration)
}

(Received 22 December 2017; published 25 January 2018)

DOI: $10.1103 /$ PhysRevLett.120.049902

In our published Letter there is an error in Fig. 2. The title of the $Y$ axis should have been "Events/day $/ 306 \mathrm{~kg} / 5 \mathrm{keV}$," where $306 \mathrm{~kg}$ was the fiducial volume. The primary message of this figure, the relative comparison of the energy spectrum, remain unchanged. To keep the $Y$-axis title as "Events/day $/ \mathrm{kg} / 5 \mathrm{keV}$ " (which is more natural), we provide a new figure. There is no change to the caption of this figure, nor any text or conclusion in the Letter.

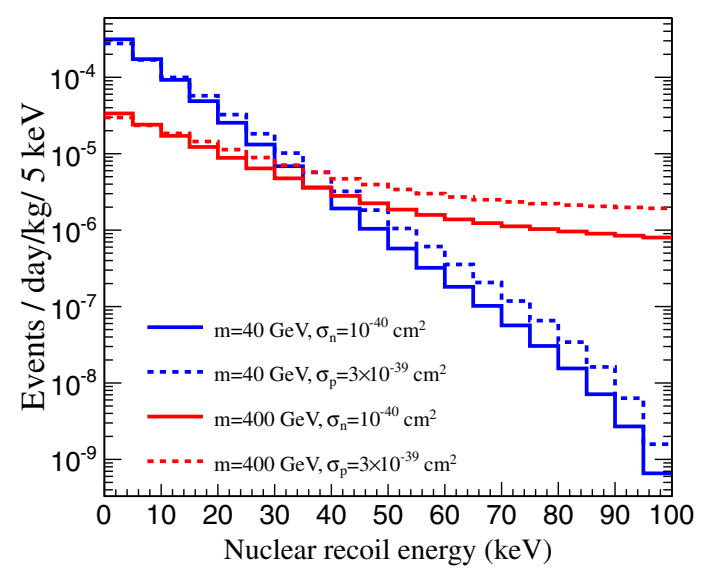

FIG. 2. Nuclear recoil-energy distributions without detector effects for two WIMP mass points 40 (blue) and $400 \mathrm{GeV} / c^{2}$ (red), for neutron-only (plain) and proton-only (dashed) couplings. Here we use $d R / d E$ calculations from Ref. [9] and structure factor calculations from Ref. [11]. The WIMP-neutron and WIMP-proton cross sections are assumed to be $\sigma_{n}=10^{-40}$ and $\sigma_{p}=3 \times 10^{-39} \mathrm{~cm}^{2}$, respectively, for visual clarity. 